\title{
Statistical Analysis of Resulting Palm vein Image through Enhancement Operations
}

\author{
Mr. Shriram D. Raut \\ Department of Computer Science and Applications \\ School of Computational Sciences \\ Solapur University, Solapur, Maharashtra, India. \\ profraut@ymail.com \\ Dr. Vikas T. Humbe \\ Department of Computer Science, \\ School of Technology, \\ S.R.T.M. University, Nanded, Sub-Campus, Latur, Maharashtra, India. \\ vikashumbe@gmail.com
}

\begin{abstract}
Nowadays biometric is playing a key role in the field of forensic and commercial applications. The vein biometrics is a robust biometric in recent trends. The vein pattern is very difficult to forge or fake. The traits are not going to be changed from birth to death. This paper discusses image enhancement operations and its result when applied on multispectral palm vein image. The image enhancement operations are much helpful to extract the vein pattern as features. The experiments can be used to highlight or trace a vein pattern lies at palm region of hand. The proposed work gains vein pattern and considered as the stepping stone towards feature extraction. The paper also discusses the comparison of statistical properties such as mean, standard deviation and coefficient of original image and images resulting out through enhancement operations. The enhancement operation is a key to gain the vein pattern. The image analysis can be framed well using statistical image measurements.
\end{abstract}

Index terms - Multispectral, histogram, feature extraction, vein pattern, near infrared, coefficient of matrix.

\section{Introduction}

Biometrics is automated methods of recognizing a person based on a physiological or behavioral characteristic; among the features measured akin to face, fingerprints, hand geometry, handwriting, iris, retinal, palm vein, and voice etc. However Palm vein biometric systems are superior because they provide a nontransferable means of identifying people not just cards or badges. A key advantage of palm vein biometric authentication is that, biometric data is based on human vein characteristics that stay constant throughout one's lifetime and are difficult to fake or change. The inspiration of our work is from Huan Zhang, Dewen $\mathrm{Hu}^{[1]}$ paper; which presents a palm vein recognition system that uses blood vessel patterns as a personal identification factor. It also discusses about an image preprocessing and feature extraction of palm vein pattern. Vein pattern identification uses an infrared light source at $760 \mathrm{~nm}$ to scan for hemoglobin in the blood ${ }^{[2]}{ }^{[6]}$. A vein pattern technology has a high degree of usability with some research showing 99.98 percent usability ${ }^{[2]}$. A palm vein is a new member of biometrics family and attracts much of the today's research attention. It has been found that light in the 700 to $1000 \mathrm{~nm}$ (infrared light) ranges can penetrate human skin, whereas $880-930 \mathrm{~nm}$ provides a good contrast ${ }^{[3]}$ of subcutaneous veins. As the palms have more complex vascular patterns than fingers and provide more distinct features for pattern matching and authentication. However with other biometric identification approaches, vein patterns are considered to be time invariant and sufficiently distinct to clearly identify an individual. The vein patterns are not easily spoofed, observed, damaged, obscured or changed and also vein pattern technology is perceived as secure as it incorporated "aliveness" detection. As per the discussion in paper the shape and texture features ${ }^{[8]}$ can be considered for parameter for image enhancement. Various enhancement operation of it can be applied on the image. It is useful to give the image analysis too. The human vascular structure is individually distinct. Even the identical twins have different and distinct vascular patterns ${ }^{[9]}$. The optical penetration depth for near-infrared imaging at $850 \mathrm{~nm}$ is estimated to be $3.57 \mathrm{~mm}$ and such illumination has shown to offer higher contrast for the subcutaneous veins while imaging ${ }^{[10]}$.

An image enhancement is one of the key stages of digital image processing. The image enhancement operations considered to be the stepping stone to perform feature extraction. It is one of the key stages of digital image processing.

This paper discusses about the usefulness of fundamental image enhancement operations. The palm vein pattern and their structure, locality at palm region of hand under subcutaneous skin are also discussed. 
These enhancement techniques are much useful to trace and highlight palm vein pattern; which lies at palm region of hand. The experiment is performed in MATLAB; which is technical computing tool to implement digital image processing techniques and operations. The paper also discusses about the statistical analysis of these resulting enhanced palm vein images with original palm vein image.

\subsection{Palm Vein Pattern}

Vein pattern identification uses an infrared light source to scan for hemoglobin in the blood. Deoxygenated hemoglobin appears as a black pattern with the hand or finger showing as a lighter color or white. The device then captures an image of vein patterns in wrist, palm, back of the hand, finger or face. This is similar to the technique used to capture retinal patterns. The backs of hands and palms have more complex vascular patterns than fingers ${ }^{[7]}$ and provide more distinct features for pattern matching and authentication.

As with other biometric identification approaches, vein patterns are considered to be time invariant and sufficiently distinct to clearly identify an individual. In this paper the biometric palm vein based recognition system is developed on the basis statistical properties of palm vein pattern. As it is found physiological biometric can be easily forged by undergoing medical operation and resulted into the medical identity loss of the person. So a strong and robust biometric feature such as palm vein can be studied and is adopted for person identification and it is very difficult to forge and is application for the living being as it monitors the blood fluctuation in the vein as heart pump. The two basic considerations in the design of a multispectral palm print system are the following:

1) The color-absorptive and color-reflective characteristics of human skin and

2) The light spectra to be used when acquiring images. Human skin is made up of three layers:

1) Epidermis; 2) dermis; and 3) sub cutis, as shown in Fig. 1. Each layer will contain a different proportion of blood and fat.

The epidermis also contains melanin, whereas the sub cutis contains veins [3]. Different light wavelengths will penetrate to different skin layers and illuminate in different spectra. NIR light penetrates human tissue further than visible light, and blood absorbs more NIR energy than the surrounding tissue (e.g., fat or melanin) [4]. The system acquires spectral information from all three dermal layers by using both visible and NIR bands. In the visible spectrum, a threemonocular LED array is used with Red peaking at 660 $\mathrm{nm}$, Green peaking at $525 \mathrm{~nm}$, and blue peaking at 470 $\mathrm{nm}$. In the NIR spectrum, an NIR LED array peaking at $880 \mathrm{~nm}$ is used.

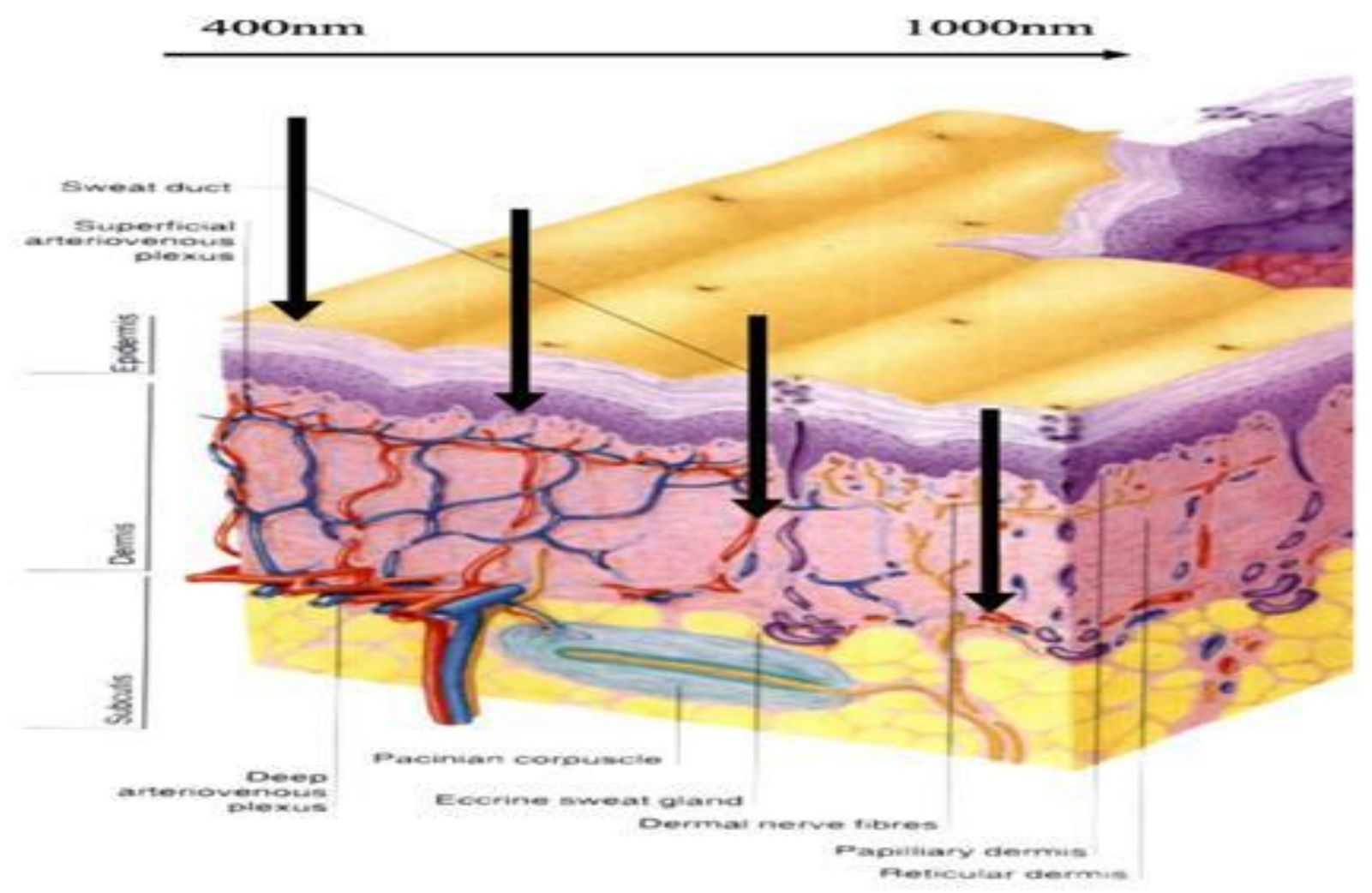

Figure 1. Cross sectional view of skin 
It has been shown that light in the 700- to $1000-\mathrm{nm}$ range can penetrate human skin, whereas $880-930 \mathrm{~nm}$ provides a good contrast of subcutaneous veins [4].

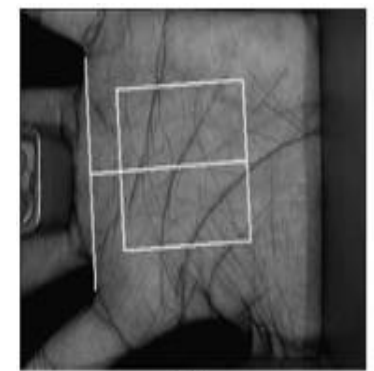

(a)

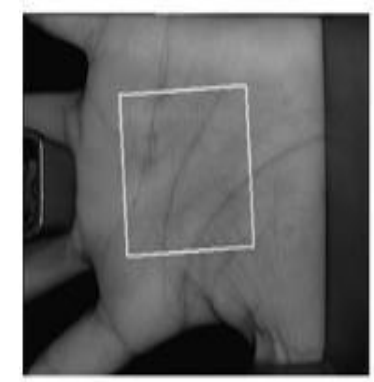

(c)

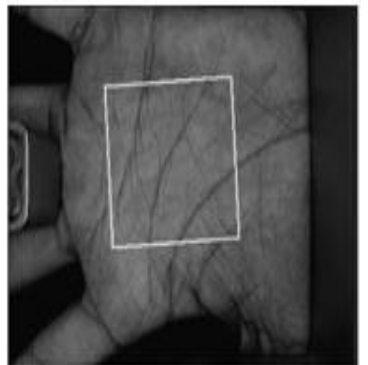

(b)

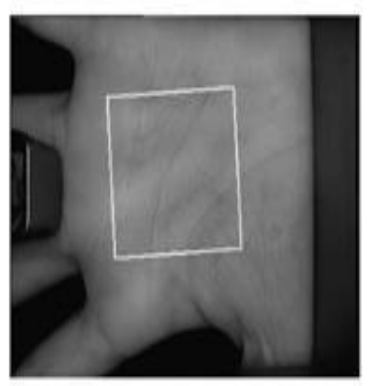

(d)
Figure 2. Typical multispectral palm print sample (a) Blue, (b) Green. (c) Red. (d) NIR. The white square is the ROI of the image.

\section{Image Database}

The Biometric Research Centre (UGC/CRC) at The Hong Kong Polytechnic University has developed a real time multispectral palm print capture device which can capture palm print images under blue, green, red and near-infrared (NIR) illuminations, and has used it to construct a large-scale multispectral palm print database ${ }^{[5]}$. Multispectral palm print images were collected from 250 volunteers, including 195 males and 55 females. The age distribution is from 20 to 60 years old.

They have collected samples in two separate sessions. In each session, the subject was asked to provide 6 images for each palm. Therefore, 24 images of each illumination from 2 palms were collected from each subject. In total, the database contains 6,000 images from 500 different palms for one illumination. The average time interval between the first and the second sessions was about 9 days. And also provide the extracted ROI images using our ROI extraction algorithm from 500 different palms for one illumination.

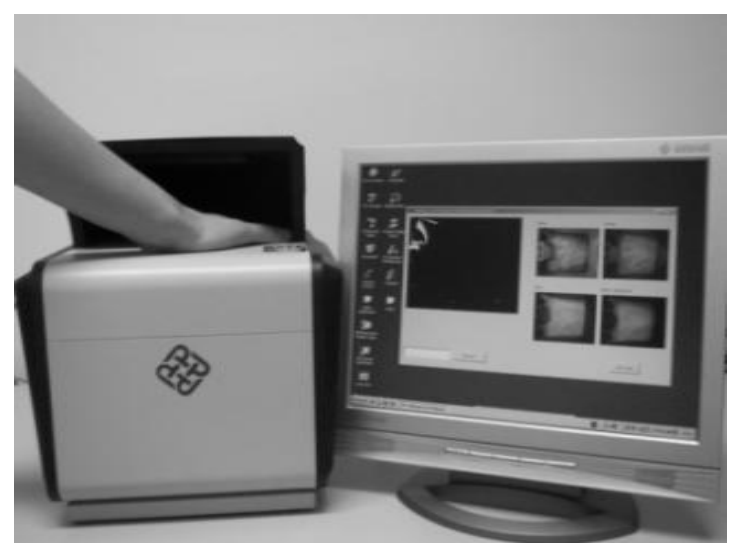

Figure 3. The outlook of multispectral palm print image acquisition device.

The average time interval between the first and the second sessions was about 9 days. Each part of database is named as "nnnn". "nnnn" represents the identity of the person (range from 1 to 500). In each folder, the first 6 images $\left(1 \_\mathrm{mm}\right)$ were captured in the first session and the latter 6 images (2_mm) were captured in the second session, "mm" represents the image index for give session (range from 1 to 6). The database contains all the original palm print images collected with our device by blue, green, red and NIR illumination ${ }^{[5]}$. In our study we have worked on the NIR illuminated palm print multispectral image and related statistical measurements. The palm-vein images in contactless imaging present lot of translational and rotational variations. Therefore, more stringent preprocessing steps are required to extract a stable and aligned ROI.

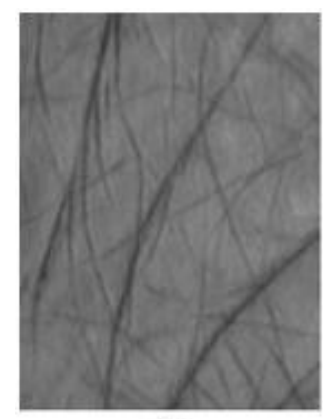

(a)

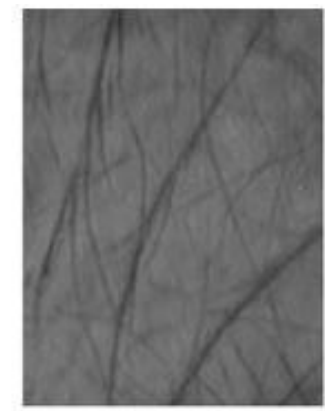

(b)

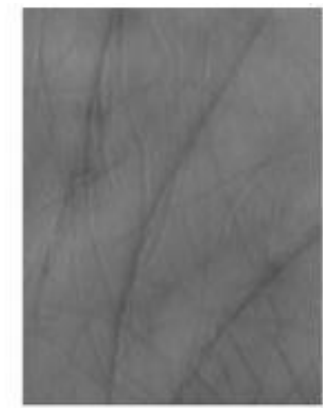

(c)

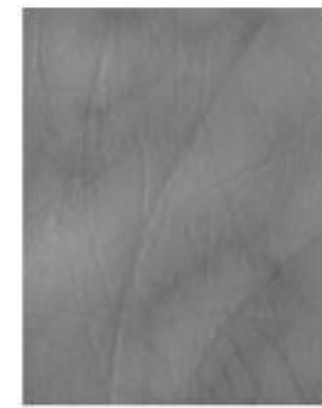

(d)

Figure 4. ROI of fig 2 (a) Blue, (b) Green. (c) Red. (d) NIR 
The preprocessing steps essentially recover a fixedsize ROI from the acquired images which have been normalized to minimize the rotational, translational, and scale changes. This is followed by the nonlinear enhancement so that the vein patterns from ROI images can be observed more clearly

\section{Image Enhancement}

The principal objective of enhancement is to process an image so that the result is more suitable than the original image for a specific application. Visual evaluation of image quality is a highly subjective process, thus making the definition of a "good image" an elusive standard by which to compare algorithm performance. Spatial domain methods are procedures that operate directly on these pixels. Spatial domain processes ${ }^{[3]}$ will be denoted by the expression:

$$
g(x, y)=T[f(x, y)]
$$

Where $f(x, y)$ is the input image, $g(x, y)$ is the processed image, and $T$ is an operator on $f$, defined over some neighborhood of $(x, y)$. The simplest form of $T$ is when the neighborhood is of size $1 \times 1$ (that is, a single pixel).In this case, $g$ depends only on the value of at $(x, y)$, and $T$ becomes a gray-level (also called an intensity or mapping) transformation function of the form $S=T(r)$.

\section{Methodology}

The following are image enhancement operations performed on image they are:

Histogram equalization

The histogram of a digital image with gray levels in the range $[0,1-1]$ is a

$$
(r k)=n k,
$$

Where rk is the $\mathrm{k}$ th gray level and rk is the number of pixels in the image. It is common practice to normalize a histogram by dividing each of its values by the total number of pixels in the image, denoted by $n$.

Thus, a normalized histogram is given by ${ }^{[11]}$ :

$$
p(r k)=\frac{n k}{n}, \text { for } k=0,1, \ldots . l-1
$$

We consider a discrete formulation and allow pixel values to be in the interval $[0,1-1]$. For any $r$ satisfying for mentioned conditions; we focus attention on transformations of the form:

$$
\begin{array}{r}
s=T(r) \\
0 \leq r \leq 1
\end{array}
$$

That produces a level $s$ for every pixel value $r$ in the original image. For reasons that will become obvious shortly, we assume that the transformation function $T(r)$ satisfies the following conditions:

(a) $T(r)$ is single-valued and monotonically increasing in the interval $0 \leq r \leq 1$ and

$$
\text { (b) } 0 \leq T(r) \leq 1 \text { for } 0 \leq r \leq 1
$$

\subsection{De-correlation Stretch}

This applies to de-correlation stretch to multichannel image.

$$
\begin{aligned}
& s=\operatorname{decorrstretch}(i) \\
& s=\operatorname{decorrstretch}(i, T O L) \\
& s=\operatorname{decorrstretch}(i),
\end{aligned}
$$

Applies a de-correlation stretch to a multichannel image $i$ and returns the result in $s$. The $s$ has the same size and class as $i$. The mean and variance in each band are the same as in $i . s=$ decorrstretch $(i, T O L)$ applies a contrast following the de-correlation stretch.

\subsection{Adaptive Histogram Equalization}

This is Contrast-limited adaptive histogram equalization (CLAHE).

$$
j=\operatorname{adapthisteq}(i)
$$

Where $\mathrm{j}=$ adapthisteq(i)enhances the contrast of the grayscale image i by transforming the values using contrast-limited adaptive histogram equalization (CLAHE).CLAHE operates on small regions in the image, called tiles, rather than the entire image. The contrast, especially in homogeneous areas, can be limited to avoid amplifying any noise that might be present and will occur in the image.

$$
j=\text { adapthisteq }(i, \text { param } 1, \text { val1,param } 2, \operatorname{val} 2 \ldots)
$$

It specifies any of the additional parameter/value.

\subsection{Adjust Map Image}

It maps the intensity values in grayscale inputted image to new values in as enhanced image such that $1 \%$ of data is saturated at low and high intensities of inputted image. This increases the contrast of the output image.

$$
j=\operatorname{imadjust}\left(i,\left[\operatorname{low}_{\text {in }} ; \text { high }_{\text {in }}\right],\left[\operatorname{low}_{\text {out }} ; \text { high }_{\text {out }}\right]\right)
$$

\subsection{Noise Removal}

The best-known order-statistics filter is the median filter, which, as its name implies, replaces the value of a pixel by the median of the gray levels in the neighborhood of that pixel: [11] 


$$
\begin{aligned}
& f^{x, y}=\operatorname{median}\{g(s, t)\} \\
& \{s, t\} \in \boldsymbol{s} \mathrm{xy}
\end{aligned}
$$

The original value of the pixel is included in the computation of the median. Median filters are quite popular because, for certain types of random noise, they provide excellent noise-reduction capabilities, with considerably less blurring than linear smoothing filters of similar size.

\section{Experiment and Results}

An experiment is carried out at MATLAB; which is software computing tool. In our experiment we have read palm vein image; which is a multispectral image captured under near infrared illumination. An experiment is focused on enhancement of image. Fundamental image enhancement operations are performed on image captured under the NIR spectrum, an NIR LED array peaking at $880 \mathrm{~nm}$ as shown in fig.4 (d). The enhancement operations are useful to extract palm vein pattern from an image.

The experimental work and image enhancement result can be summarized as follows-

1. When we apply adjust color map operation then vein pattern and principal lines are quite visible.

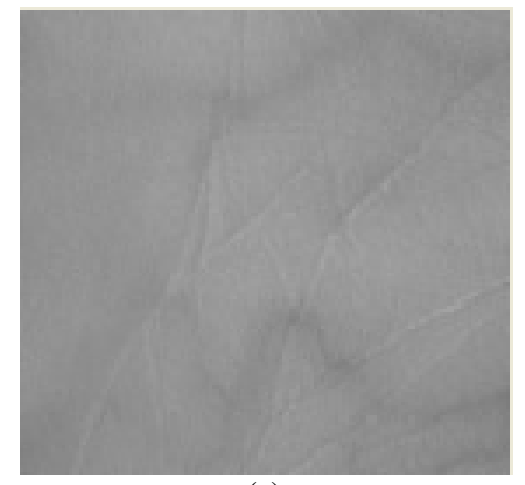

(a)

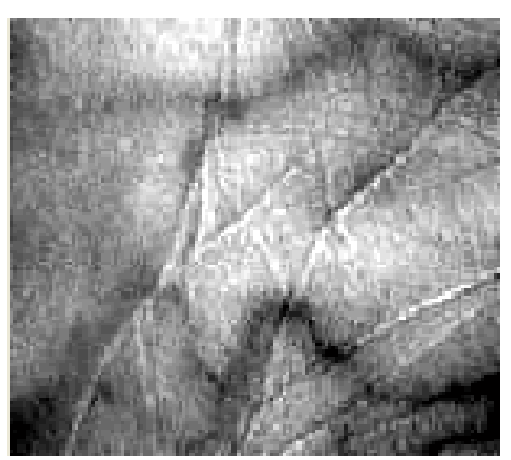

(b)

Figure 5. (a) Original image; (b) color map adjusted image.
2. A de-correlation stretch image enhancement can be performed. An image resulting has a dark shaded region i.e. actually vein pattern

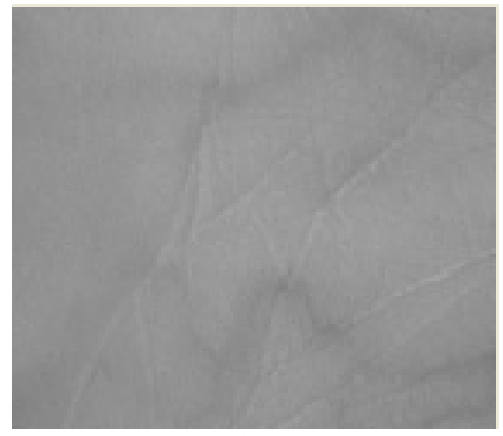

(a)

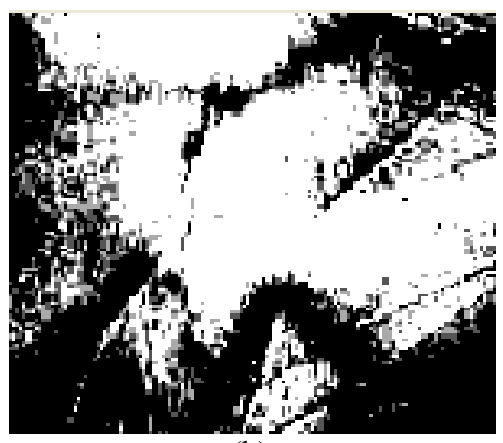

(b)

Figure 6. (a) Original image; (b) visually enhanced image.

3. We have enhanced contrast of original image using histogram equalization.

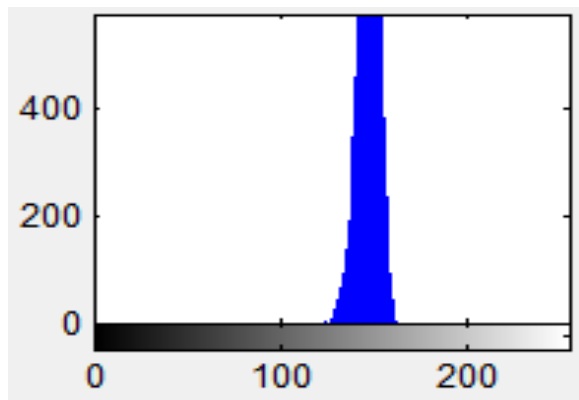

(a)

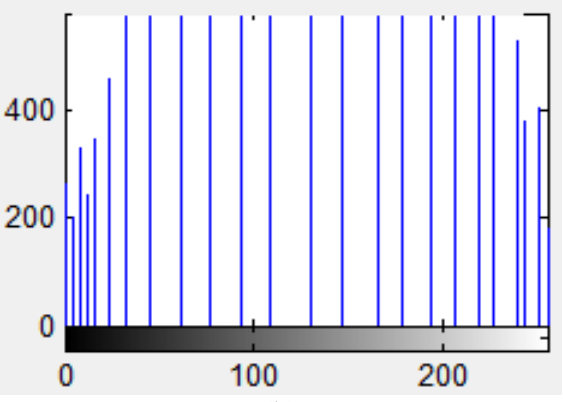

(b) 


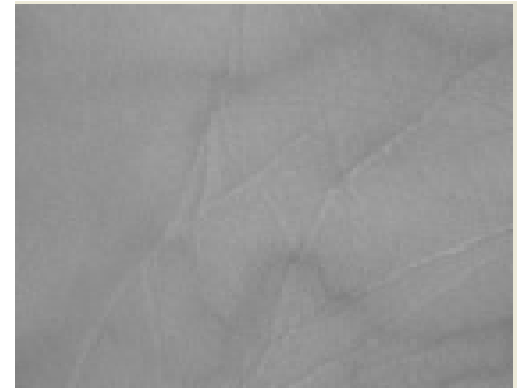

(c)

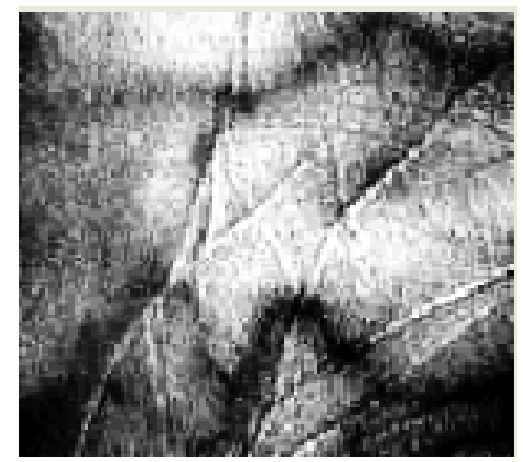

(d)

Figure 7. (a) Histogram of original; (b) equalized histogram; (c) original image; (d) enhanced image.

4. The contrast limited adaptive histogram equalization (CLAHE) is performed on an image.

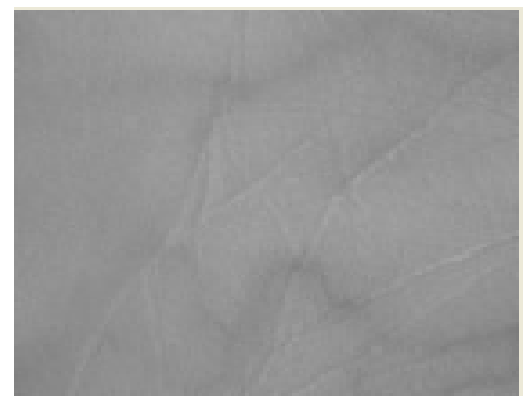

(a)

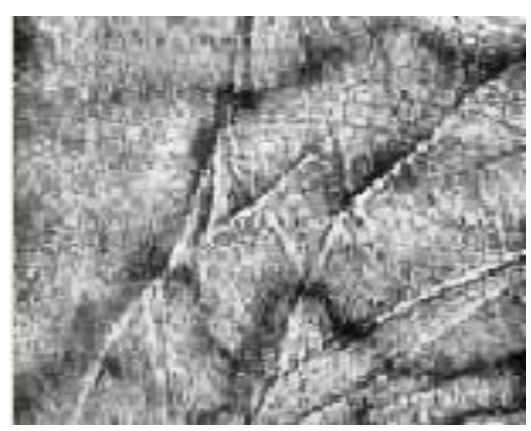

(b)

Figure 8. (a) Original image; (b) CLAHE resultant image.

5. Apply contrast stretch operation by specifying lower and upper limits that can be used for contrast stretching image.

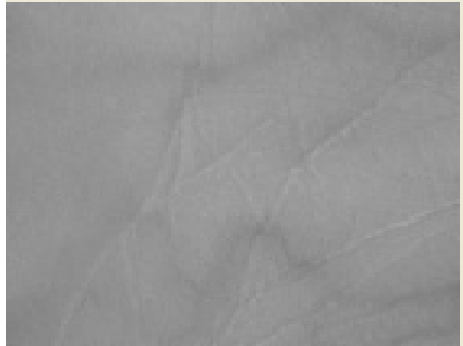

(a)

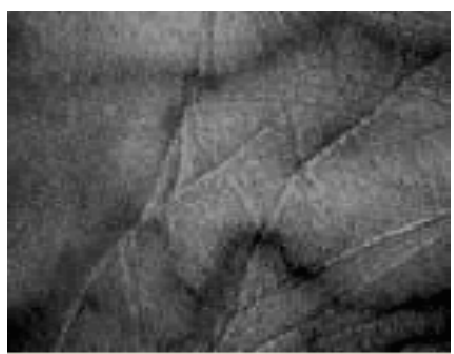

(b)

Figure 9. (a) Original image; (b) Contrast stretched resulting image.

\section{Comparison}

As per the table 1; which we can state that there is consistently decrease in the mean value of original image and image enhanced through enhancement operations. The analysis on the basis mean can be concluded as the mean value of contrast limited adaptive histogram equalized image is high and contrast stretched image has the lowest mean value as compared to other enhancement operations. The standard deviation is next parameter of consideration for comparison. There is much difference in the standard deviation value of original image and enhanced image.

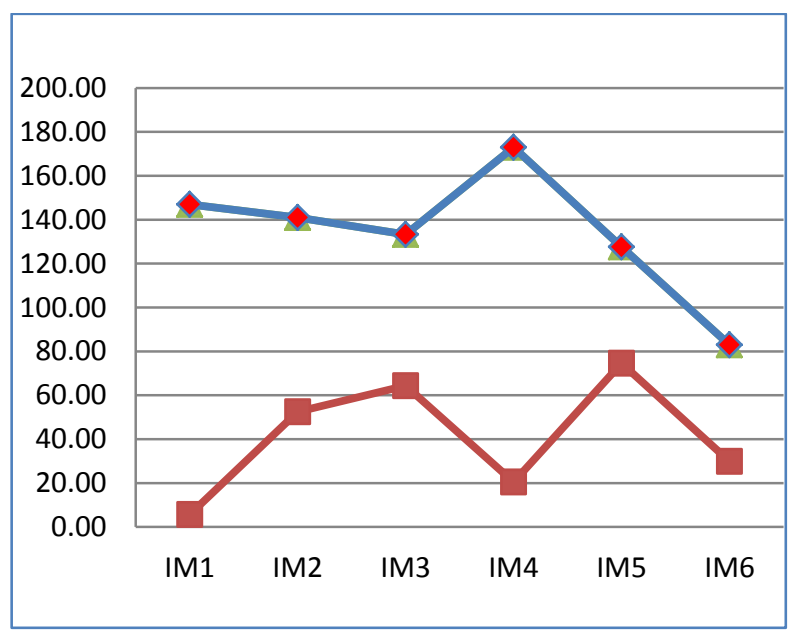

Figure 10. Plot of mean and standard deviation values of the original image with respect to enhanced images; as per table 1. 
We find image enhanced by histogram equalization is having higher standard deviation value and with at maximum difference compared to original image. With every image enhancement operation we find standard deviation is most varying statistical value with much difference from one another. The fig. 10 shows a plot of the mean and standard deviation values of each original and enhanced palm vein image. This value varies from one another. The values are found to be unique and distinct.

The third factor of comparison is correlation of images. The correlation coefficient is calculated by considering original image with every enhanced image, means it's a ratio of original image and enhanced image. There is bit change of values of original image with enhanced image. The correlation coefficient value of color map adjusted image is much high and image as result of contrast limited adaptive histogram equalization is much low compared to the values of other enhancement operations. The values are in between 0 and 1 but are very fuzzy values. These values are also varies from one another. From this analysis we can conclude that statistical values for every operation are distinct and show an effective result of enhancement operation over original image.

\section{Conclusion}

A palm vein is a new member of pattern recognition and biometrics family, has attracted much of the research attention. De-oxygenated hemoglobin appears as a black pattern with the hand or finger showing as a lighter color or white. The image preprocessing operations such as image enhancement are performed. The result shows how fundamental image enhancement operations are useful so as to trace or highlight the vein pattern that lies at palm of hand. The result also shows enhancement in an image that shows palm features such vein structure as well as principal lines. The analysis is framed by taking into consideration the statistical values of original image and enhanced image through enhancement operations. These features are useful for pattern matching or simply classification of an individual. So, the objective of experiment is successful and leads to extract the palm vein pattern from a multispectral image captured under near infrared illumination; which are not easily spoofed, observed, damaged, obscured or changed and also vein pattern technology is perceived as secure as it incorporated "aliveness" detection.

TABLE 1. THE COMPARISON BASED ON STATISTICAL VALUES OF ORIGINAL IMAGE AND ENHANCED IMAGE.

\begin{tabular}{|c|c|c|c|c|c|c|}
\hline & $\begin{array}{c}\text { Original } \\
\text { Image } \\
\text { (IM1) }\end{array}$ & $\begin{array}{c}\text { Color } \\
\text { map } \\
\text { adjusted } \\
\text { image } \\
\text { (IM2) }\end{array}$ & $\begin{array}{c}\text { de- } \\
\text { correlation } \\
\text { stretch } \\
\text { image } \\
\text { (IM3) }\end{array}$ & $\begin{array}{c}\text { Contrast } \\
\text { limited } \\
\text { adaptive } \\
\text { histo.equal. } \\
\text { (IM4) }\end{array}$ & $\begin{array}{c}\text { histogra } \\
\mathrm{m} \\
\text { equalized } \\
\text { image } \\
\text { (IM5) }\end{array}$ & $\begin{array}{c}\text { contrast } \\
\text { stretched } \\
\text { Image } \\
\text { (IM6) }\end{array}$ \\
\hline Mean & 146.9181 & $\begin{array}{c}141.013 \\
1\end{array}$ & 133.3055 & 172.9891 & 127.6122 & 82.9482 \\
\hline $\begin{array}{c}\text { Standard } \\
\text { Deviation }\end{array}$ & 5.6380 & 52.4362 & 64.3818 & 20.5105 & 74.5520 & 29.8759 \\
\hline $\begin{array}{c}\text { Correlatio } \\
\mathrm{n}\end{array}$ & & 0.9985 & 0.9914 & 0.7785 & 0.9744 & 0.9984 \\
\hline
\end{tabular}

\section{Acknowledgment}

We would like to thank the Research Centre at Hong Kong Polytechnic University as they provided an access to their image database. We are also grateful to University Department of Computer Science and Applications, School of Computational Sciences, Solapur University, Solapur and University Department of Computer Science, School of Technology, S.R.T.M.U.N. Sub-Centre, Latur for providing us an infrastructure and research laboratories.

\section{References}

[1] Huan Zhang, Dewen Hu. A Palm Vein Recognition System [J]. International Conference on Intelligent Computation Technology and Automation, Vol. 1, pp.285-288, IEEE Computer Society, 2010.

[2] Ishani Sarkar1, Farkhod Alisherov, Tai-hoon Kim, and Debnath Bhattacharyya. A Palm vein Authentication: Review [J]. International Journal of Control and Automation, Vol. 3, No. 1, 2010.

[3] D. J. Gawkrodger. Dermatology: An Illustrated Colour Text. [M]. 3rd ed. Elsevier Health Sci., 2002. 
[4] V. P. Zharov, S. Ferguson, J. F. Eidt, P. C. Howard, L. M. Fink, and M. Waner. Infrared imaging of subcutaneous veins [A]. Lasers Surg. Med., vol. 34, no. 1, pp. 56-61, Jan. 2004.

[5] David Zhang, Zhenhua Guo, Guangming Lu, Lei Zhang, Wangmeng Zuo. An Online System of Multispectral Palm prints Verification [J]. IEEE Transactions on Instrumentation and Measurement, Vol. 59, No. 02, pp.480-490, 2010.

[6] D. M. Mancini, L. Bolinger, H. Li, K. Kendrick, B. Chance and J. R. Wilson. Validation of Nearinfrared Spectroscopy in Humans [J]. Journal of Applied Physiology, Vol. 77, no. 06, pp. 27402747, 1994.

[7] N Miura, A. Nagasaka, and T. Miyatake. Extraction of Finger-Vein Patterns Using Maximum Curvature Points in Image Profiles [J]. Journal of Machine Vision Application, pp. 347350, Volume E90-D, Issue 8, 2007.

[8] M.Deepamalar, M.Madheswaran. An Enhanced Palm Vein Recognition System Using Multi-level Fusion of Multimodal Features and Adaptive Resonance Theory [J]. International Journal of Computer Applications, Vol. 1-No. 20, pp.95-10, 2010.

[9] Li Xueyan, Guo Shuxu. Fourth Biometric-Vein Recognition. Pattern recognition techniques, technology and applications [B]. ISBN -953-761924-4, 2008.

[10]A. Kumar and K. V. Prathyusha. Personal authentication using hand vein triangulation $[\mathrm{J}]$. IEEE Trans. Image Process. vol. 38, no. 9, pp. 2127-2136, Sep. 2009.

[11] Rafael C. Gonzalez. Digital Image Processing [B]. Second Edition, University of Tennessee Richard E. Woods, MedData Interactive.

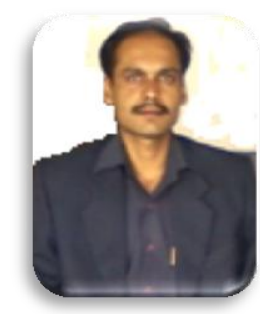

Dr. Vikas T. Humbe has completed his Ph.D. degree from University Department of Computer Science and Information Technology, Dr.B.A.M.U. Aurangabad and working as the Assistant Professor at Department of Computer Science, School of Technology, S.R.T.M. University, Nanded, Subcampus, Latur. In teaching, he has been focusing on Digital Image and video processing concepts and Problem Based Learning approaches in Computer Science Education. In research, his current interests include Pattern Recognition, Image and video based processing, Data ware housing and web mining etc. He has published 42 research articles at National/International conferences and journals also he is the author of the books. He has published 13 Journals and 21 papers in National and International Conferences and also working as reviewer for various International and National Journals and Conferences like Elsevier's Pattern Recognition Letters, Journal on Machine Vision and Applications, Academic Journals, IEEE IJCNN 07 and 09, ACVIT-09 etc. He is Member of IACSIT Singapore, Member of IAEng, Hong Kong, CSTA, USA and Graduate Member of IEEE USA. His area of research interest is Biometrics, Image Processing, Computer Vision and Video Processing. $\mathrm{He}$ is the IEEE Graduate Member and has immense research recognition worldwide.

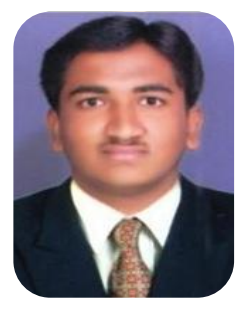

Mr. Shriram D. Raut is pursuing his Ph.D. degree in Computer Science, and research scholar. He has completed his M.Sc. degree in Computer Science from University Department of Computer Science and Information Technology from Dr.B.A.M.U. Aurangabad and is working as the Assistant Professor at Department of Computer Science and Application, School of Computational Sciences, Solapur University, Solapur. He worked as the Research Scholar and submitted a project in Computer Science under the UGC SAP (II) DRS Phase-I: 2009-2014 under the theme "Biometric: Multimodal System Development". 